\title{
Design of Multifunctional Anti-Theft Backpack Based on Microcomputer Liping Wang ${ }^{1, a^{*}}$, Fei $\mathrm{Xu}^{1, \mathrm{~b}}$ and Tuanbu Wang ${ }^{1, \mathrm{c}}$ \\ ${ }^{1}$ School of Information Engineering, Xijing University, Xi'an 710123,China \\ a wlpggg@163.com, b107803350 @qq.com, c597669319 @qq.com
}

Keywords: MCU; Anti-Theft; APP; Infrared Sensor; Alarm

\begin{abstract}
In this paper, a multifunction anti-theft backpack is designed. The control system uses a micro controller as the control core powered by battery. It can be set different working modes by the remote control or the APP operation of the mobile phone. The system uses infrared sensor to measure the changes of the human information in the external environment, and it combines the signal of the zipper sensor to control the alarm circuit to work. The multifunction anti-theft backpack has the basic function of the common backpack. At the same time, it has high anti-theft and robbing function, and it also has the functions of mobile charging and automatic lighting inside the bag. The multifunction anti-theft backpack is more convenient and comfortable.
\end{abstract}

\section{Introduction}

In modern society, most people go out with backpack is almost a daily. How to ensure the security of the things in the backpack in public is a matter for each of us. With the improvement of people's living standards, personal awareness of the protection of private property has been increasing. Therefore, higher requirements for backpack security are required. The traditional backpack is only a simple facility. No matter how reasonable the design is, how strong the material is, how novel it is, the thefts can open it through a variety of means. Now there are many kinds of burglar backpacks in the market, but most of them are made in the bag material and zipper. The anti-theft backpack's anti-theft performance is limited and the safety factor is not high. There are also backpacks using wireless communication technology, cryptography technology to strengthen its anti-theft performance. These backpacks have tedious control circuit ,high cost and large volume, and they use inconveniently for users. So, it is only used in some suitcases, and it is less used in the backpack that is carried in daily travel. Moreover, with the improvement of people's living standard, there are new requirements for the convenience, comfort and other aspects of backpacks.

\section{The Structure Composition of the Control System}

Functions of Multifunctional Anti-Theft Backpack. The anti-theft function is the basic function of the multi-function anti-theft backpack. The control circuit selects the pyroelectric infrared sensor. It converts the infrared signal of the human body into the electrical signal in non contact way. At the same time, the zipper position sensor is used to detect the zipper state. The sensors send the detection signal to the controller which controls the alarm circuit work. The backpack control circuit is powered by a mobile power supply, and different modes of operation can be set by controlling the operation of the remote control. At the same time, backpack also has the function of mobile charging, automatic lighting inside the bag. The multi-function anti-theft and anti robbery backpack has the basic functions of the ordinary backpack, and has high anti-theft and anti robbery functions, and is more convenient and comfortable in using.

The functions and use instructions are shown as follows:

(1) In the anti-theft mode, when the hand reaches into the backpack, the backpack sends out the alarm sound.

(2) When the backpack is robbed, the backpacker can press alarm button of the portable remote control, then the backpack can send out the sound and light alarm signal, The loud alarm can make the robbers afraid to escape abandoning the bag.

(3) The backpack is internally provided with LED lights, and LED is automatically lit when the 
zipper in open state.

(4) In the heating mode, heating plate work to heating mildly.

The Structure Composition of Control Circuit. The backpack control circuit is composed of six modules: microcomputer control module, human infrared induction module, zipper position induction module, alarm circuit module, working mode setting module and LED lighting module. The system control unit selects the STC15 single chip microcomputer, and selects the micro human infrared induction module PM-6 in real time whether the hand is entered into the backpack illegally. The zipper position sensor is selected to detect the zipper opening and closing state, so as to achieve the alarm purpose.

The block diagram of the control circuit is shown in figure 1.

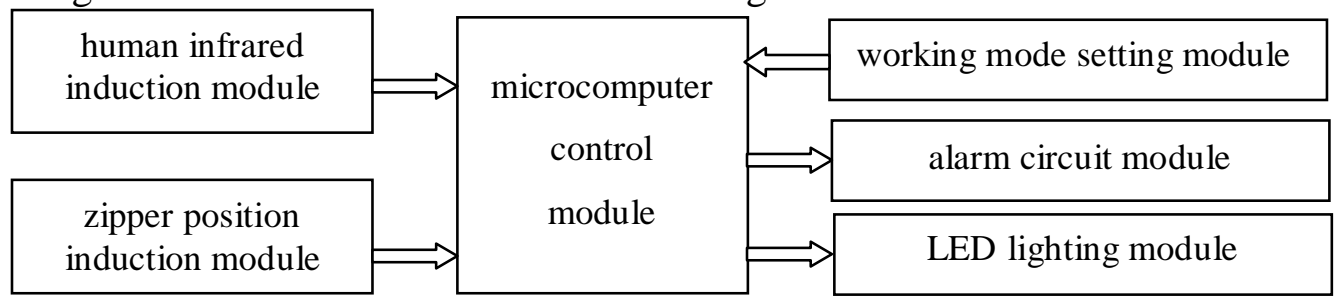

Figure 1. Structure composition of control circuit

The system structure block diagram shows that the main control single chip computer is the center. The infrared sensor of the human body detects the infrared signal of the human body and send it to the single chip microcomputer. The zipper switch sensor is used to judge the zipper switch state through the movement of the magnet position. The alarm information is sent to alarm circuit after processing in microcomputer unit. The LED lighting module is directly controlled by the SCM program, and the LED illuminate automatically when the zipper is opened in normal state. The working mode of the whole system is set by ordinary portable remote controller.

\section{Hardware design of control system}

Power Supply Module. Considering that many people go out a mobile charging cable in the present time, so the power supply uses a conventional rechargeable mobile power supply, and there are multiple external USB standard interfaces. The mobile power unit can provide power to the whole control circuit of the backpack, and it meets the charging needs of various mobile devices in the market.

Controller Module. The circuit selects SIC15 single chip as the control core. It is a low power and high performance CMOS 8 bit microcontroller. The minimum system circuit of the microcomputer is shown in Figure 2. The minimum system of single chip is composed of crystal oscillator and reset circuit. The crystal oscillator produces the clock frequency required by the single chip microcomputer. The reset circuit is used to recovery the circuit to the starting state.

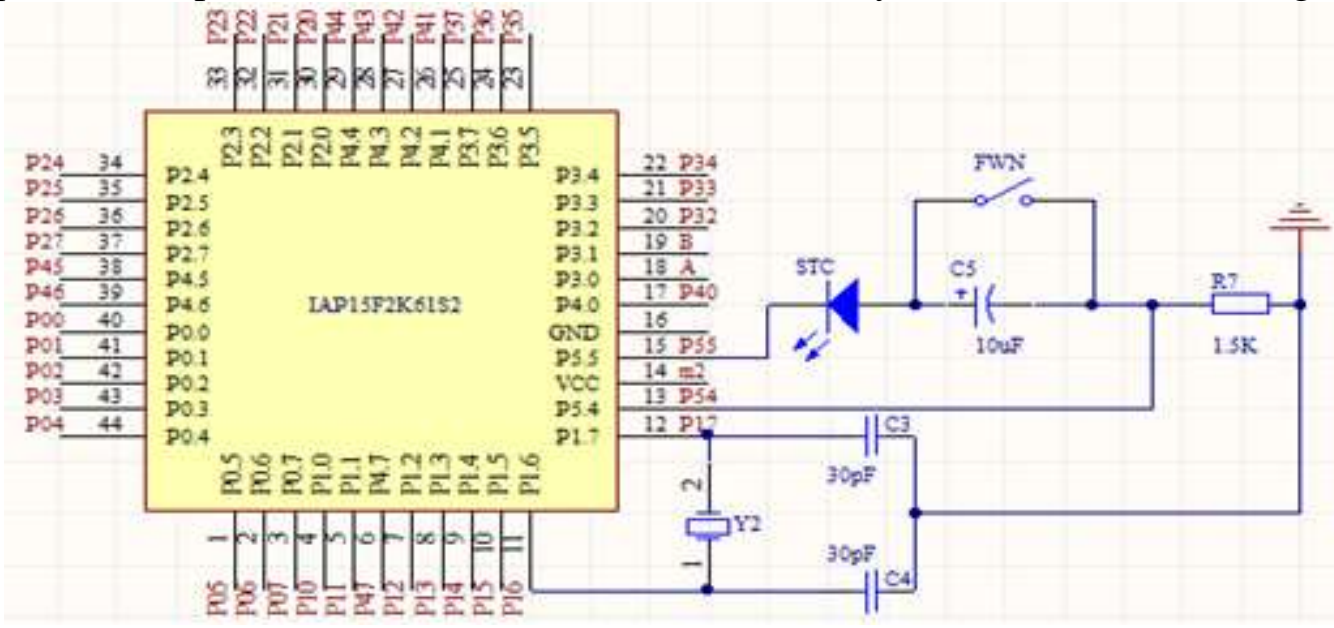

Figure 2. Minimum system of single chip microcomputer

Work Mode Setting Module. The control circuit of backpack can be set different working modes by the remote control or the APP operation of the mobile phone. It is in the lighting state 
automatically when it is powered. The system use infrared sensor to measure the changes of the human information in the external environment, and it combine the signal of the zipper sensor to control the alarm circuit to work. The working mode setting module is composed of two parts of the transmitting module and the receiving module, which can be set by the portable remote control device or the mobile phone APP directly. The whole system is completed by the MCU controller.

In the design of multi-function intelligent anti-theft backpack, there are four working modes.

MODE1: mode 1 (lighting), the hand enters the package to be bright, does not alarm, does not care about the zipper switch state;

MODE 2: mode 2 (anti-theft), open the zipper or hand into the bag to alarm immediately.

MODE 3: mode 3 (alarm), unconditional alarm;

MODE 4: mode 4 (heating), heating switch, press change heating state.

The work mode is set through the remote control or the mobile phone APP, press the key on the remote control, the receiver receives the corresponding signal to the single chip computer and sets the different working mode.

Infrared Sensing Module. The circuit use a miniature human infrared induction module PM-6 to induce the human body signal. It is a kind of micro power and suitable for the use of mobile power supply. It is very convenient to dock with various kinds of circuits. It has a full automatic induction function which outputs high level when human body in its range of induction. After the controller receives the signal, it notifies the alarm circuit and sends out the sound and light alarm signal. As long as the person leaves its induction range, the circuit automatically outputs low level after a few seconds.

The external circuit of the miniature human infrared induction module is simple. Its pin connection diagram is shown in Figure 3: pin 1 is signal terminal; pin 2 is positive power terminal; pin 3 is negative power terminal.

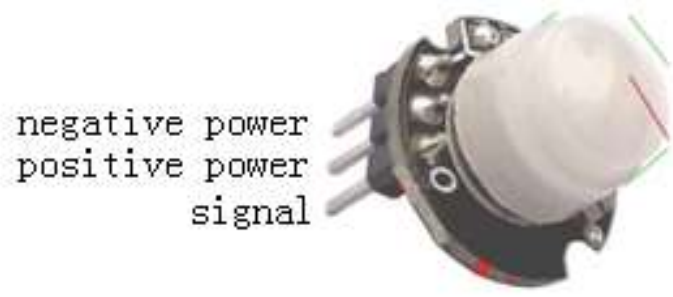

Figure 3. Miniature human infrared induction module PM-6

Zipper Position Sensing Module. The zipper switch induction module is operated by a small volume contact switch reed pipe and magnet. The reed pipe is simple in structure and fast in operation, and has a normally open or normally closed contact form. In this module, the normally open contact is selected. When the bag is in normal state that the zipper is not opened and the magnet is far away from the dry reed pipe, the reed pipe is in a state of disconnection. When the bag zipper is pulled away, the magnet is close to the dry reed pipe and the reed is magnetized. Then the contact is closed, and the buzzer gives out the alarm sound. When the magnet is away from the dry reed pipe, the reed will break the contact by its own elasticity.

Alarm Module. In order to consider the possibility of encountering a sudden special situation, the alarm circuit also adds a manual alarm function in addition to the automatic alarm. In the normal operation of the circuit, even if the sensor circuit and the optical control circuit do not work, the backpacker can also carry out the manual alarm. Pressing the switch of the remote control, the alarm unit starts to work immediately and the alarm state continues. When the B button of the remote control is pressed, the relay coil is out of power and the alarm unit stops working.

Lighting and Heating Module. The LED lighting module adopts high brightness LED lamp, which has smaller size and higher brightness. It can completely meet the needs of the illumination inside the package. The heating module uses a nonwoven heating sheet, which is a flexible electric heating film. Its structure is a sandwich structure whose upper and lower surfaces are made up of a non-woven fabric with a high temperature resistance and excellent insulation. The intermediate heating body is a very durable, special hot wire that can withstand folding. Because the working current of the heating plate is large, it can not be directly connected with the single chip 
microcomputer. So the heating module is connected with the single chip microcomputer through the relay.

\section{Software Design of Control System}

The system software is written by Keil C, and the software is simulated and debugged with Proteus software. Finally, the actual circuits installation test is carried out.

The remote control has four buttons, which are K1, K2, K3 and K4. Four buttons correspond to four working states of MODE1, MODE2, MODE3 and MODE4. It use variable C1 show the state of the infrared sensor. When $\mathrm{C} 1=1$, it indicates that the hand is in the package. It use variable $\mathrm{C} 2$ show the state of the zipper position sensor. When $\mathrm{C} 2=1$, it indicates that the zipper is opened.

When the control circuit works, four working states are set up first. In the normal state, the backpack lighting module runs, which makes it convenient for us to take the things in the bag. When the backpack is in the state of security, the states of that whether the hand is in the bag or whether the zipper is opened determine the operation of the alarm module. The operation of the alarm module can be stopped when the $\mathrm{K} 1, \mathrm{~K} 2$ or $\mathrm{K} 4$ key is pressed. When the backpack is in the state of anti-robbery, pressing the K3 key can start the alarm module immediately. Pressing K4 key can set the backpack into the heated mode, and then the heating strip on the back of the backpack work to heat slowly.

\section{Conclusion}

The multi-function intelligent anti-theft backpack not only has the ordinary function, but also realize the anti-theft and anti-robbing functions. It can be set different working modes through the remote control or APP operation. Using the infrared sensor, it measure the external environment factors and then control the alarm circuit work. At the same time, the backpack also has the function of mobile charging, automatic lighting inside the bag, and back heating and heating. Backpack has a higher function of burglar and guard against theft. It is easy to use and comfortable,s and has strong practical value and promotion value.

\section{References}

[1] He Bin. STC microcontroller C language programming [M], Tsinghua University press, Beijing, 2016.

[2] Fan Jingyan. Application of sensors and detection technology [M], Machinery Industry Press, Beijing, 2005.

[3] Lai Qingmin. Interface and example of sensor and MCU [M], Aerospace University Press, Beijing, 2008.

[4] Jiang Bin, Xu Jianliang. A zero intermediate frequency direct sequence spread spectrum digital receiver technology $[\mathrm{J}]$. Electronic design engineering, 2015 (16): 68-70.

[5] Zuo Wei. Overview of the development and key technologies of HF communication system[J]. Communication technology ,2014(8):847-853.

[6] Xin Dengsong. Shortwave communication technology and communication frequency selective [J]. Chinese summarized new communication, 2016(4):33-34.

[7] Wang Jinlong. Research and practice of HF Digital Communication [M]. Beijing: Science Press, 2013.

[8] Han Xiaoming. Design of emergency communication system based on HF communication technology [J]. Chinese new communications, 2016(8):11-14.

[9] Jiang Hongkui, Liu Min, Kim. A new scheme of 8PSK shortwave communication technology of [J]. Electronic technology, 2016(8):84-86.

[10] Zhang Huike, Xu Songyi, Shi Liu. Application of M-ary orthogonal spread spectrum technique in scattering communication [J]. Radio communication technology, 2015 (2):82 - 84. 